\title{
Galectin-9 ameliorates fulminant liver injury
}

\author{
TOMOKO TADOKORO $^{1}$, ASAHIRO MORISHITA $^{1}$, TEPPEI SAKAMOTO $^{1}$, SHINTARO FUJHHARA $^{1}$, \\ KOJI FUJITA ${ }^{1}$, SHIMA MIMURA ${ }^{1}$, KYOKO OURA ${ }^{1}$, TAKAKO NOMURA ${ }^{1}$, JOJI TANI ${ }^{1}$, HIROHITO YONEYAMA ${ }^{1}$, \\ HISAKAZU IWAMA ${ }^{2}$, TAKASHI HIMOTO ${ }^{3}$, TOSHIRO NIKI ${ }^{4}$, MITSUOMI HIRASHIMA ${ }^{4}$ and TSUTOMU MASAKI ${ }^{1}$ \\ ${ }^{1}$ Department of Gastroenterology and Neurology and ${ }^{2}$ Life Science Research Center, Faculty of Medicine, Kagawa University, \\ Kagawa 761-0793; ${ }^{3}$ Department of Medical Technology, Kagawa Prefectural University of Health Sciences, Kagawa 761-0123; \\ ${ }^{4}$ Department of Immunology and Immunopathology, Faculty of Medicine, Kagawa University, Kagawa 761-0793, Japan
}

Received April 12, 2016; Accepted March 16, 2017

DOI: $10.3892 / \mathrm{mmr} .2017 .6606$

\begin{abstract}
Fulminant hepatitis is a severe liver disease resulting in hepatocyte necrosis. Galectin-9 (Gal-9) is a tandem-repeat-type galectin that has been evaluated as a potential therapeutic agent for various diseases that regulate the host immune system. Concanavalin A (ConA) injection into mice results in serious, immune-mediated liver injury similar to human viral, autoimmune and fulminant hepatitis. The present study investigated the effects of Gal-9 treatment on fulminant hepatitis in vivo and the effect on the expression of microRNAs (miRNAs), in order to identify specific miRNAs associated with the immune effects of Gal-9. A ConA-induced mouse hepatitis model was used to investigate the effects of Gal-9 treatment on overall survival rates, liver enzymes, histopathology and miRNA expression levels. Histological analyses, TUNEL assay, immunohistochemistry and miRNA expression characterization, were used to investigate the degree of necrosis, fibrosis, apoptosis and infiltration of neutrophils and macrophages. Overall survival rates following ConA administration were significantly higher in Gal-9-treated mice compared with control mice treated with ConA + PBS. Histological examination revealed that Gal-9 attenuated hepatocellular damage, reduced local neutrophil infiltration and prevented the local accumulation of macrophages and liver cell apoptosis in ConA-treated mice. In addition, various miRNAs induced by Gal-9 may contribute to its anti-apoptotic, anti-inflammatory and pro-proliferative effects on hepatocytes. The results of the present study
\end{abstract}

Correspondence to: Dr Asahiro Morishita, Department of Gastroenterology and Neurology, Faculty of Medicine, Kagawa University, 1750-1 Ikenobe, Miki-cho, Kita-gun, Kagawa 761-0793, Japan

E-mail: asahiro@med.kagawa-u.ac.jp

Abbreviations: Gal-9, galectin-9; miRNAs, microRNAs; ConA, concanavalin A

Key words: galectin-9, fulminant hepatitis, microRNA demonstrate that Gal-9 may be a candidate therapeutic target for the treatment of fulminant hepatitis.

\section{Introduction}

Fulminant hepatitis remains a major health problem worldwide, and this condition may occur from multiple origins, including viral hepatitis, autoimmune hepatitis, drug-induced liver injury, metabolic disease and circulatory disturbances (1). Immune-mediated mechanisms appear to have a central role in acute hepatitis, however, detailed mechanisms have not previously been elucidated $(2,3)$. Due to the lack of specific treatments for fulminant hepatitis, plasma exchange is usually provided to patients with this condition (1). In addition, liver transplantation, usually from living donors, is scheduled when clinical improvement is not achieved with these conventional medical treatments $(1,4)$.

Concanavalin A (ConA) injections in mice result in serious, immune-mediated liver injury similar to that observed in human viral, autoimmune and fulminant cases of hepatitis $(5,6)$. ConA injection results in an increase in the serum concentrations of several inflammatory cytokines, including tumor necrosis factor- $\alpha$, interleukin- 6 and interferon- $\gamma$, which contribute to the development of hepatitis (5-7). In the present study, a ConA-induced mouse hepatitis model was used to investigate the effectiveness of galectin-9 (Gal-9) as a novel therapeutic target in fulminant hepatitis. Gal-9 is a $\beta$-galactoside-binding lectin belonging to the galectin family, and was first identified as an eosinophil chemoattractant and activation factor (5). Gal-9 has been previously reported to induce apoptosis in T cells, particularly in CD 4 + Th1 and Th17 cells, and this lectin additionally exhibits a stimulatory effect on regulatory T cell activity (6-8). Notably, Gal-9 has been investigated as a potential therapeutic agent for various autoimmune diseases (8). Furthermore, Gal-9 has previously been demonstrated to exhibit anti-allergenic (9) and anti-tumor (10) effects. However, there are few reports demonstrating the effects of Gal-9 in fulminant hepatitis.

miRNAs are small, non-coding RNAs, and their number within the human genome is predicted to be $\sim 1,000$ (11). There are $>2,000$ genes encoding human miRNAs that are registered in miRBase (http://www.mirbase.org) (12). It has been demonstrated that the expression of miRNAs is associated 
with various liver diseases, including hepatitis and hepatocellular carcinoma (13-15). However, miRNA expression profiles following Gal-9 administration in fulminant hepatitis remain to be elucidated. Therefore, the present study aimed to investigate if Gal-9 ameliorates fulminant hepatitis and to identify specific miRNAs associated with the effects of Gal-9 in hepatitis.

\section{Materials and methods}

Mice and treatments. Animal experiments were performed according to the guidelines of the Animal Care and Use Committee for Kagawa University (Takamatsu, Japan). Ethical approval was obtained from the Animal Care and Use Committee for Kagawa University. A total of 56 male BALB/c mice, at 7 weeks of age, 21-26 g, were purchased from Japan SLC, Inc. (Hamamatsu, Japan). The mice were housed together in a temperature-controlled environment under a $12 \mathrm{~h}: 12 \mathrm{~h}$ day:night cycle (light from 07:00 to 19:00) at $22 \pm 1^{\circ} \mathrm{C}$ with freely available food and water throughout the experiment. Mice were given a single intravenous injection of ConA (Merck KGaA, Darmstadt, Germany) at a dose of $35 \mathrm{mg} / \mathrm{kg}$ body weight, and the animals were sacrificed $24 \mathrm{~h}$ following ConA administration or were observed for $48 \mathrm{~h}$ while checking for survival every $6 \mathrm{~h}$. In the Gal-9 treated group $(\mathrm{n}=17), 00 \mu \mathrm{g}$ Gal-9 (Department of Immunology, Faculty of Medicine, Kagawa University, Takamatsu, Japan) per mouse was injected subcutaneously immediately following the administration of ConA. Controls were treated with only PBS $(n=10)$, only Gal-9 $(n=10)$ or PBS with ConA $(n=19)$.

Analysis of liver enzymes in mice. Alanine aminotransferase (ALT) activities were measured by L-Type AST.J2 (Wako Pure Chemical Industries, Ltd., Osaka, Japan) and analyzed by an enzymatic colorimetric test (Bio Majesty JCA-BM8060; JEOL Ltd., Tokyo, Japan) according to Japan Society of Clinical Chemistry transferable method (16).

Histological analyses. Livers and spleens from individual mice were fixed for $\sim 48 \mathrm{~h}$ in $10 \%$ formalin at room temperature $\left(22 \pm 1^{\circ} \mathrm{C}\right)$. Tissue samples were dehydrated by ethanol and xylene. After embedding in paraffin wax, sections with a thickness of $5 \mu \mathrm{m}$ were cut by Shikoku Cytopathological Laboratory (Takamatsu, Japan) and stained with hematoxylin and eosin to determine the degree of necrosis. Image analysis of the necrotic area was performed using a light microscope, (BX51; Olympus Corporation, Tokyo, Japan) with cellSens version 1.14 (Olympus Corporation). The sections of livers were also stained using the Masson Trichrome staining procedure (Merck KGaA), according to the manufacturer's protocol, to examine the progression of fibrosis.

Terminal deoxynucleotidyl-transferase-mediated dUTP nick end labeling (TUNEL) assays. To investigate levels of apoptosis, paraffin-embedded sections $(5 \mu \mathrm{m})$ also fixed in $10 \%$ formalin as above, were stained by TUNEL assays using the TACS ${ }^{\circledR} 2$ TdT-DAB In Situ Apoptosis Detection kit (Trevigen, Inc., Gaithersburg, MD, USA), according to the manufacturer's protocol.
Immunohistochemistry. Liver paraffin sections also fixed in $10 \%$ formalin $(5 \mu \mathrm{m})$ were incubated in $3 \% \mathrm{H}_{2} \mathrm{O}_{2}$ in $20 \%$ methanol for $10 \mathrm{~min}$ to remove endogenous peroxidase activity. The sections were then treated with $2.5 \%$ normal goat serum for $30 \mathrm{~min}$ and incubated with rat anti-Ly-6G (ab25377; 1:100; Abcam, Cambridge, UK) and rabbit anti-CD11b (ab75476; 1:200; Abcam) primary antibodies in $2.5 \%$ normal goat serum overnight at $4^{\circ} \mathrm{C}$. Sections were subsequently incubated with ImmPRESS ${ }^{\mathrm{TM}}$ Reagent HRP Anti-Rat IgG (MP-7404, ready-to-use) and ImmPRESS ${ }^{\mathrm{TM}}$ Reagent Anti-Rabbit IgG (MP-7401, ready-to-use) secondary antibodies (both from Vector Laboratories, Inc., Burlingame, CA, USA), and a DAB Peroxidase (HRP) Substrate kit (Vector Laboratories, Inc.). Counterstaining were performed by Mayer's hematoxylin solution (Wako Pure Chemical Industries, Ltd.) to stain nuclei. Positive cells were counted in 10 high-power fields/section using a light microscope, at a magnification of $x 400$.

Global miRNA expression profiling. Total RNA was extracted from mouse liver samples with the miRNeasy Mini kit (Qiagen, Inc., Valencia, CA, USA), according to the manufacturer's protocol. Following the quality and quantitation of RNA measurement with an RNA 6000 Nano kit (Agilent Technologies, Inc., Santa Clara, CA, USA), the samples (250 ng RNA/sample) were labeled using a miRCURY LNA ${ }^{\mathrm{TM}}$ microRNA Hi-Power Labeling kit (Hy3; Exiqon A/S, Vedbaek, Denmark) according to the manufacturer's protocol and subsequently hybridized onto a 3D-Gene ${ }^{\circledR}$ mouse miRNA Oligo chip (version 19; Toray Industries, Inc., Tokyo, Japan) according to the manufacturer's protocol. Scanning was performed with the 3D-Gene ${ }^{\circledR}$ Scanner 3000 (Toray Industries, Inc.). The 3D-Gene ${ }^{\circledR}$ extraction version 1.2 software (Toray Industries, Inc.) was used to read the raw intensities of the image. To determine changes in miRNA expression between Gal-9-treated and control samples, the raw data was analyzed using GeneSpring GX software, version 10.0 (Agilent Technologies, Inc.).

Statistical analysis. All analyses were performed using GraphPad Prism version 6.0 for Windows (GraphPad Software, Inc., La Jolla, CA, USA). Survival rate was analyzed using the log-rank test. Unpaired comparisons between groups were performed using the Mann-Whitney U test. Differentially expressed miRNAs were also determined with the Mann-Whitney U test. Hierarchical clustering was performed using the farthest neighbor method employing the absolute uncentered Pearson's correlation coefficient as a metric. A heat map was produced using the relative expression intensity for each miRNA, in which the base-2 logarithm of the intensity was median centered for each row. $\mathrm{P}<0.05$ was considered to indicate a statistically significant difference and data are presented as mean \pm standard deviation.

\section{Results}

Gal-9 prolongs overall survival in ConA-treated mice. Notably, in ConA-treated mice, the overall survival rate was significantly increased in Gal-9-treated mice compared with control mice treated with ConA + PBS ( $\mathrm{P}<0.05$; Fig. 1). No deaths were observed in the PBS-only and Gal-9-only-treated groups. 


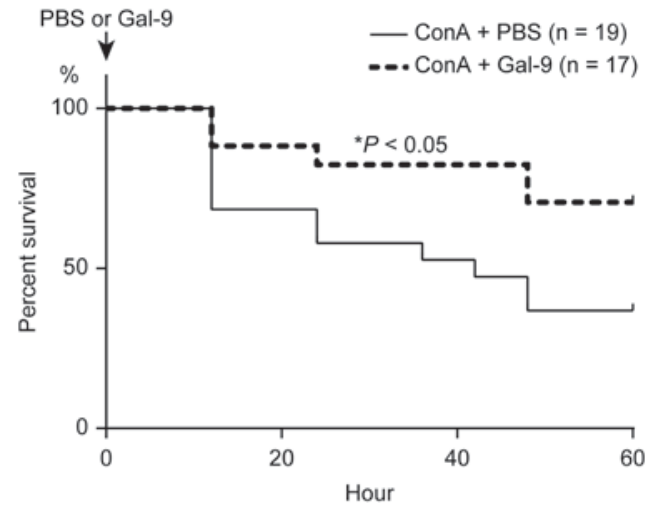

Figure 1. Overall survival rates in ConA-treated mice in presence or absence of Gal-9. Mice were given a single intravenous injection of ConA $(35 \mathrm{mg} / \mathrm{kg}$ body weight) and were observed for $48 \mathrm{~h}$ and checked for survival every $6 \mathrm{~h}$. Gal-9 (100 $\mu \mathrm{g}$ per mouse) was injected subcutaneously immediately following the injection of ConA. The overall survival rate was significantly higher in Gal-9-treated mice compared with ConA-treated control mice. ${ }^{*} \mathrm{P}<0.05$ vs. control. Con A, concanavalin A; Gal-9, galectin-9.

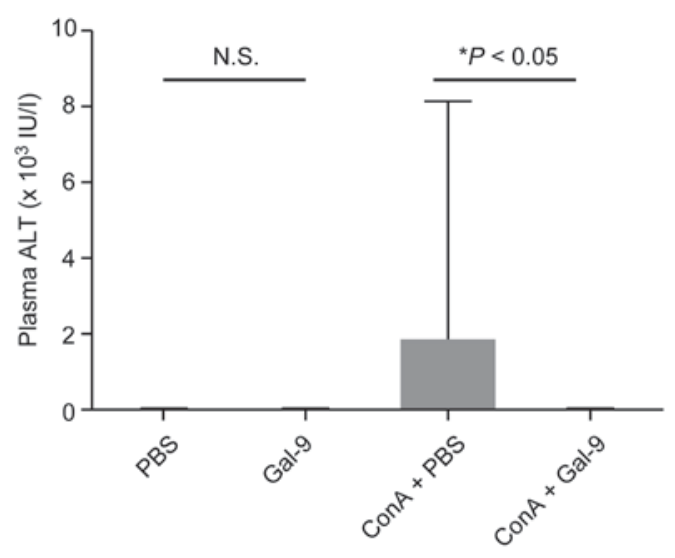

Figure 2. Effects of Gal-9 on plasma liver enzyme levels. Plasma ALT levels were measured $24 \mathrm{~h}$ following treatment. No significant alteration in plasma ALT levels (within normal limits, $<42 \mathrm{U} / 1$ ) was observed between PBS-only and Gal-9-only groups. ALT levels were markedly diminished in mice treated with ConA + Gal-9 compared with those treated with ConA + PBS. Data are presented as the mean \pm standard deviation. Gal-9, galectin-9; ALT, alanine aminotransferase; ConA, concanavalin A; N.S., not significant.

Gal-9 attenuates liver injury in ConA-treated mice. To determine the effects of Gal-9 on severe liver injury, liver enzymes were examined in the plasma of each group. Plasma ALT levels were significantly diminished in ConA-treated mice co-treated with Gal-9 compared with ConA-treated mice co-treated with PBS. No significant alteration in plasma ALT levels (within normal limits, <42 U/l) was observed between PBS-only andGal-9-only groups(Fig.2).Administration of ConA induced necrosis in the liver and congestion of blood in the spleens of ConA + PBS control mice. However, treatment in both the Gal-9 only and ConA + Gal-9 groups resulted in internal organs that were almost normal. Histologically, the necrotic areas in the livers of ConA + Gal-9-treated mice were significantly smaller compared with the ConA + PBS group $(\mathrm{P}<0.05$; Fig. 3). In addition, no fibril formation was detected in either of these groups using Masson's trichrome staining (Fig. 4A).
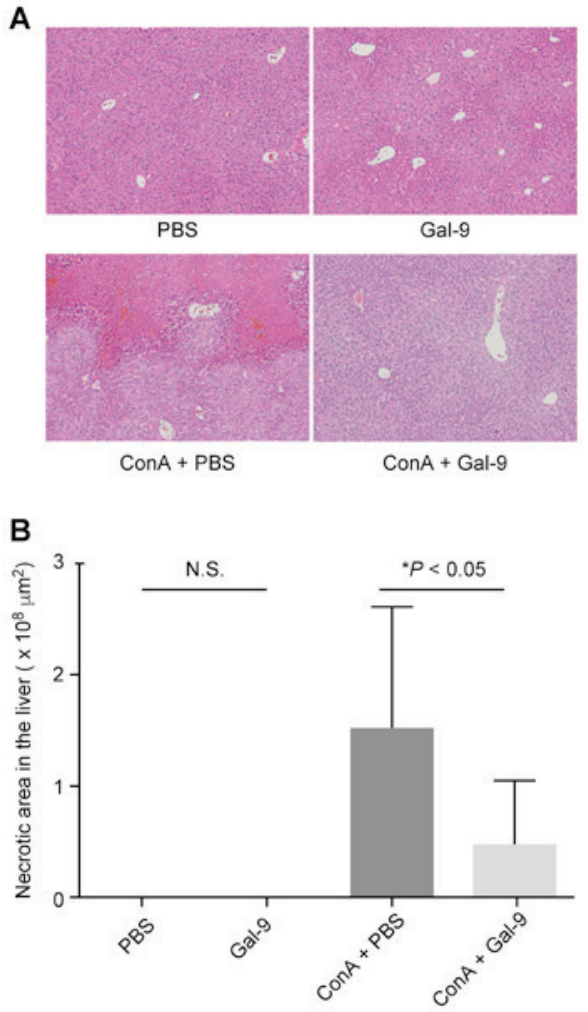

Figure 3. Gal-9 attenuates liver injury. (A) Paraffin-embedded sections were stained with hematoxylin and eosin. Original magnification, x100. (B) Image analysis of the necrotic area. No necrosis was observed in the PBS-only and Gal-9 only groups. Gal-9 significantly attenuated liver necrosis in ConA-treated mice. Data are presented as the mean + standard deviation. Gal-9, galectin-9; ConA, concanavalin A; N.S., not significant.

\section{A}
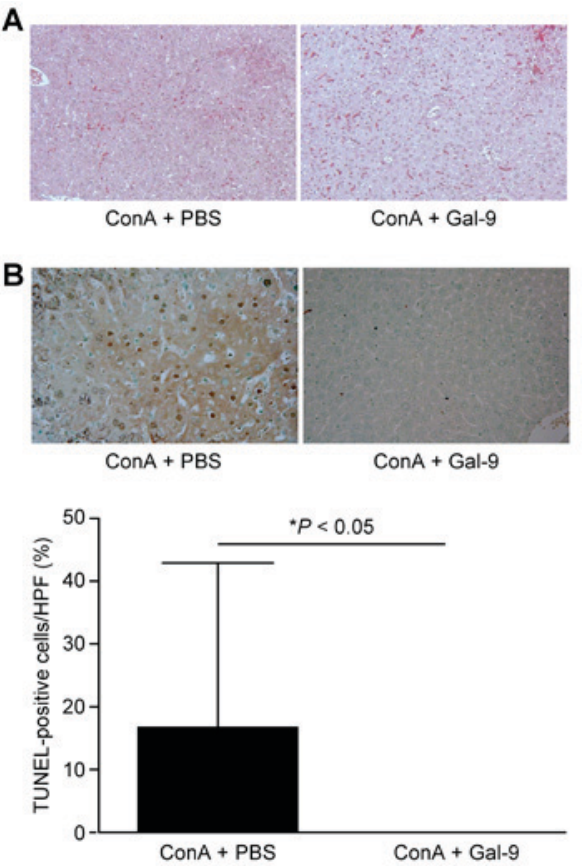

Figure 4. Gal-9 prevents the apoptosis of hepatocytes in ConA-treated mice. (A) No fibril formation was detected in either group by Masson's trichrome staining. Original magnification, x100. (B) Liver sections were stained using the TUNEL assay and the percentage of apoptotic hepatocytes was calculated. Original magnification, x200. No apoptotic cells were observed in the Gal-9 group. A decreased percentage of TUNEL-positive cells was observed in the Gal-9-treated group compared with the control group. Gal-9, galectin-9; ConA, concanavalin A. 
Table I. Alterations in miRNA expression in liver cells from mice treated with ConA in presence or absence of Gal-9.

A, Upregulated miRNAs

\begin{tabular}{lccc}
\hline miRNA & P-value & Fold-change, Gal-9/control & Chromosomal localization \\
\hline mmu-miR-598-5p & 0.002 & $2.507 \pm 1.188$ & $8 \mathrm{p} 23.1$ \\
mmu-miR-1982-5p & 0.019 & $1.823 \pm 0.293$ & - \\
mmu-miR-760-5p & 0.020 & $1.777 \pm 0.840$ & $1 \mathrm{p} 22.1$ \\
mmu-miR-133c & 0.044 & $1.704 \pm 0.808$ & - \\
mmu-miR-365-3p & 0.002 & $1.636 \pm 0.116$ & - \\
mmu-miR-361-3p & 0.034 & $1.613 \pm 0.227$ & Xq21.2 \\
mmu-miR-873a-5p & 0.006 & $1.576 \pm 0.743$ & $9 \mathrm{p} 21.1$ \\
mmu-miR-6405 & 0.016 & $1.533 \pm 0.731$ & - \\
mmu-miR-341-5p & 0.041 & $1.519 \pm 0.721$ & - \\
mmu-miR-706 & 0.038 & $1.513 \pm 0.063$ & 14 \\
mmu-miR-544-3p & 0.033 & $1.363 \pm 0.644$ & - \\
\hline
\end{tabular}

B, Downregulated miRNAs

\begin{tabular}{lccc}
\hline miRNA & P-value & Fold change, Gal-9/control & Chromosomal localization \\
\hline mmu-miR-718 & 0.045 & $0.564 \pm 0.016$ & $\mathrm{X}$ \\
mmu-miR-1899 & 0.018 & $0.576 \pm 0.275$ & - \\
mmu-miR-6335 & 0.042 & $0.621 \pm 0.071$ & - \\
mmu-miR-127-3p & 0.041 & $0.629 \pm 0.140$ & $14 \mathrm{q} 32.2$ \\
mmu-miR-181b-5p & 0.009 & $0.633 \pm 0.299$ & - \\
mmu-miR-331-5p & 0.042 & $0.634 \pm 0.304$ & $12 \mathrm{q} 22$ \\
mmu-miR-3961 & 0.041 & $0.676 \pm 0.047$ & - \\
mmu-miR-1981-3p & 0.008 & $0.728 \pm 0.344$ & - \\
mmu-miR-669d-5p & 0.030 & $0.732 \pm 0.106$ & 9 \\
mmu-miR-3091-3p & 0.044 & $0.749 \pm 0.116$ & - \\
mmu-miR-142-5p & 0.025 & $0.755 \pm 0.042$ & $17 \mathrm{q} 22$ \\
mmu-miR-346-5p & 0.029 & $0.763 \pm 0.086$ & $10 \mathrm{q} 23.2$ \\
\hline
\end{tabular}

Fold change is presented as the mean \pm standard deviation. '-'=chromosomal location unclear. miR, miRNA; miRNA, microRNA; ConA, concanavalin A; Gal-9, galectin-9.

Gal-9 prevents apoptosis in ConA-treatedmice. Apoptotic cells were detected by TUNEL assay in the livers of ConA-treated mice, with or without Gal-9 co-treatment. Although apoptotic cells were observed in the livers without Gal-9 treatment, as illustrated in Fig. 4B, fewer apoptotic cells were detected in Gal-9-treated mice $(\mathrm{P}<0.05) ; 16.5 \%$ of hepatocytes were TUNEL-positive per field in the livers of mice without Gal-9 treatment (Fig. 4B).

Gal-9 reduces local neutrophil and macrophage infiltration. In ConA-induced hepatitis, the present study performed immunohistochemical staining for Ly-6G and CD11b expression. Gal-9 prevented neutrophil (Ly-6G; P<0.05; Fig. 5A) and CD11b-positive macrophage $(\mathrm{P}<0.05$; Fig. 5B) infiltration compared with the ConA + PBS control group.

miRNA expression profiles in ConA-treated mouse livers with or without Gal-9 co-treatment. As presented in Table I, 11 miRNAs were significantly upregulated $24 \mathrm{~h}$ following Gal-9 treatment, whereas 12 miRNAs were significantly downregulated $(\mathrm{P}<0.05$; Table I). Unsupervised hierarchical clustering analysis using Pearson's correlation demonstrated that Gal-9-treated mice clustered separately from the control mice (Fig. 6).

\section{Discussion}

Fulminant hepatitis has various origins, including viral hepatitis, autoimmune hepatitis, drug-induced liver injury, metabolic disease and circulatory disturbances (1). Novel treatments for chronic hepatitis, including hepatitis $\mathrm{B}$ and $\mathrm{C}$, have been developed and have been demonstrated to be effective (17-19). However, the current treatments for fulminant hepatitis are inadequate. For example, antiviral therapy is not sufficient for the treatment of fulminant hepatitis $B$, as this virus is not directly cytopathic and the mechanisms of fulminant hepatitis B have been suggested to be primarily immune-mediated (20). Currently, no specific treatment is available for fulminant hepatitis and therefore novel curative immunological approaches are critical for fulminant hepatitis therapy. 


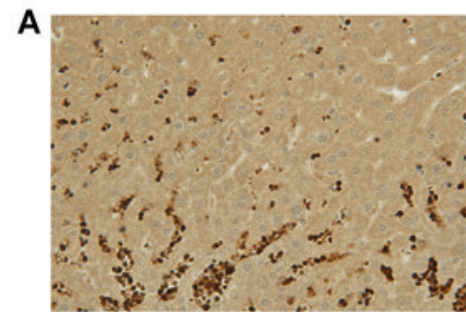

ConA + PBS

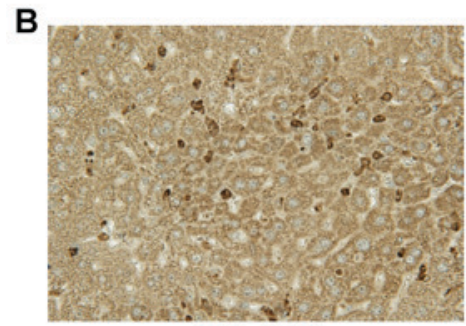

ConA + PBS

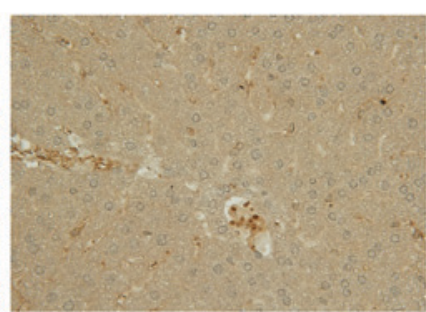

ConA + Gal-9

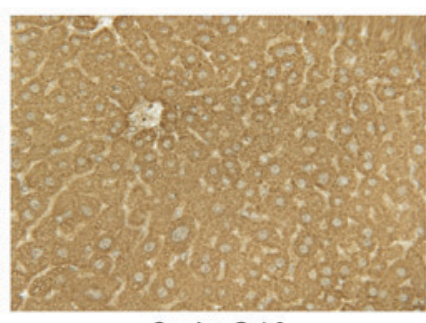

ConA + Gal-9
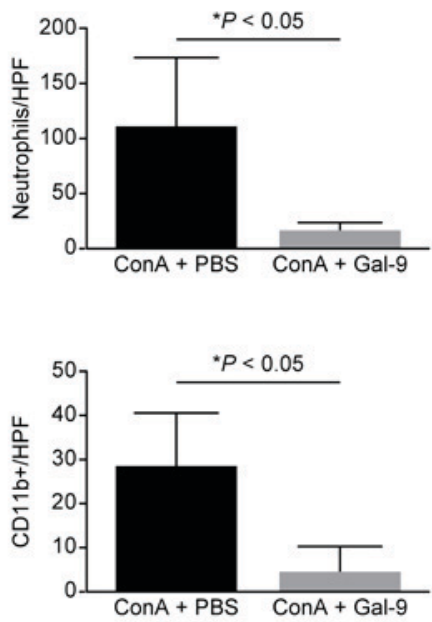

Figure 5. Gal-9 prevents the accumulation of neutrophils (Ly-6G) and macrophages (CD11b) in ConA-induced hepatitis. (A) Left, immunohistochemistry of neutrophils in representative liver sections. Original magnification, x200. Right, quantification of neutrophils per HPF (magnification, x400). (B) Left, immunohistochemistry of macrophages in representative liver sections. Original magnification, x200. Right, quantification of macrophages per HPF (magnification, $\mathrm{x} 400)$. The infiltration of neutrophils and macrophages in Gal-9 treated mice was reduced compared with control animals. Data are presented as the mean + standard deviation. Gal-9, galectin-9; ConA, concanavalin A; HPF, high power field.

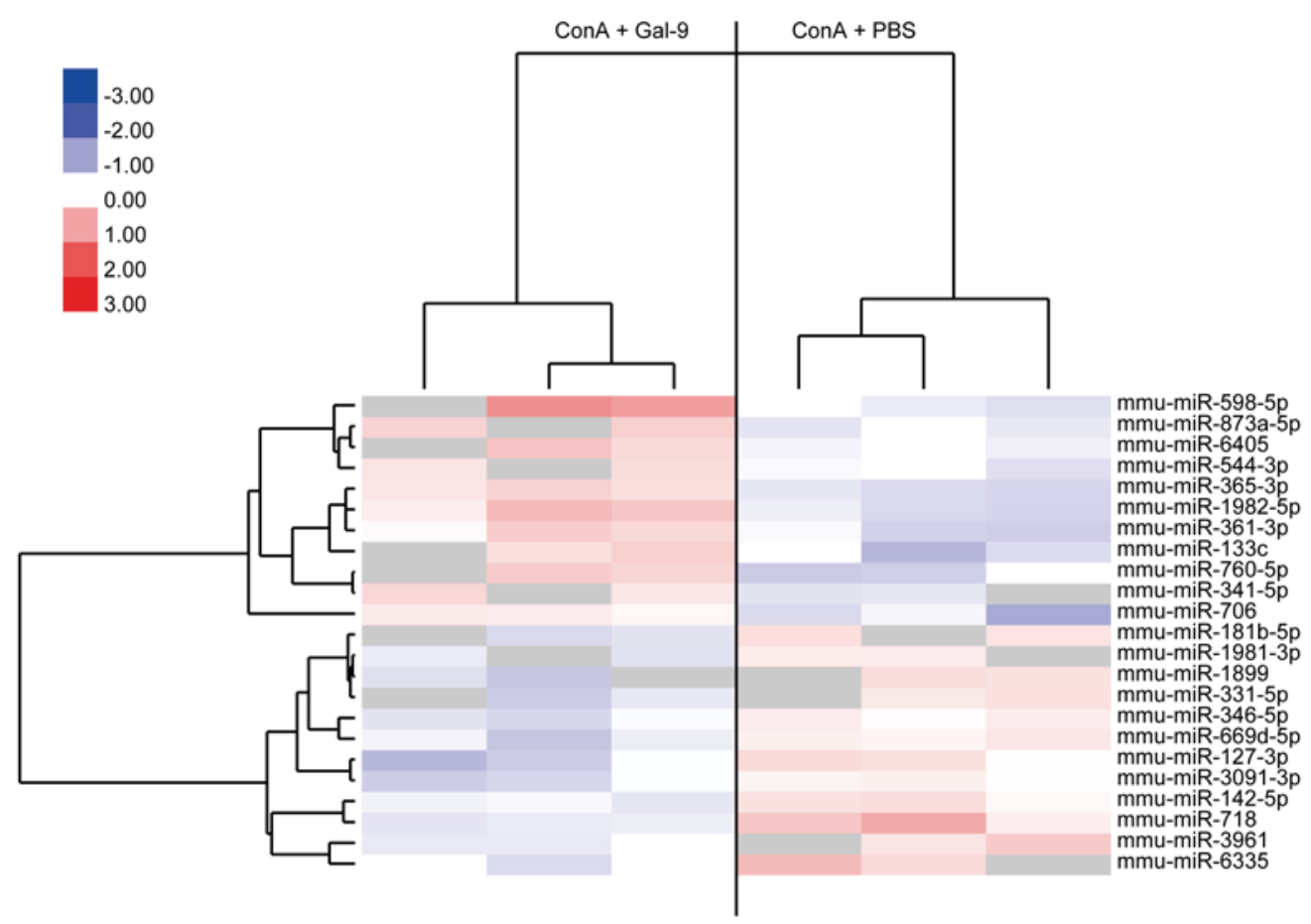

Figure 6. Hierarchical clustering of ConA-treated mice with and without Gal-9. Liver tissue was clustered according to the expression profiles of differentially expressed miRNAs between ConA-treated mice with and without Gal-9. The analyzed samples are reported in columns and the miRNAs are presented in rows. The miRNA clustering tree is presented on the left and the sample clustering tree appears at the top. The color scale at the top indicates the relative expression levels of miRNAs, with red representing high expression levels and blue representing low expression levels. ConA, concanavalin A; Gal-9, galectin-9; miR, miRNA; miRNA, microRNA.

The present study demonstrated that a single injection of Gal-9 improved the survival rates of ConA-treated mice. Gal-9 treatment also reduced local neutrophil and macrophage infiltration. Gal-9 is a ligand of T cell immunoglobulin and mucin protein 3 (6), and it induces Th1 cell apoptosis, thereby inhibiting Th1 immunity and leading to peripheral tolerance (21). Furthermore, Gal-9 results in selective apoptosis of ConA-activated CD4-positive T cells and ameliorates ConA-induced hepatitis (22). These reports indicate that Gal-9 may regulate immune responses to induce apoptosis in T cells.

In the current study, fewer apoptotic hepatocytes were observed in livers treated with Gal-9 compared with those 
not treated with Gal-9. Indeed, it has been reported that Gal-9 suppresses hepatocyte apoptosis via the inhibition of toll-like receptor 4 expression in ischemia-reperfusion injury $(23,24)$. Hepatocyte apoptosis was previously demonstrated to be significantly increased in hepatitis $(25,26)$, including viral hepatitis and nonalcoholic steatohepatitis, and has been demonstrated to be associated with disease severity $(25,26)$. These results indicate that Gal-9 may suppress hepatocyte apoptosis even in severe liver injury.

Notably, Gal-9 induces cancer cell apoptosis and inhibits the proliferation of various human cancer cell types, including melanoma (10), hepatocellular carcinoma (27) and cholangiocarcinoma (28). The fact that Gal-9 suppresses hepatocyte apoptosis in liver injury and induces cancer cell apoptosis appears paradoxical. However, Gal-9 may alter its mechanism of action depending upon the situation and the necessity to maintain hepatic homeostasis.

The present study identified miRNAs associated with the effects of Gal-9 in fulminant hepatitis, and miR-361 and miR-133 were upregulated in the livers of Gal-9-treated mice compared with control mice. Kanitz et al (29) reported vascular endothelial growth factor (VEGF) A as a putative target of miR-361. In addition, miR-361 regulates endothelial progenitor cell functioning by targeting VEGF (30). VEGF is a potent stimulator of angiogenesis and also contributes to inflammation via plasma extravasation (31). Furthermore, miR-133, which was also upregulated by Gal-9 in the current study, exhibits anti-apoptotic effects by targeting caspase-9 (32) and enhances myoblast proliferation by repressing serum response factor (12). Therefore, upregulation of miR-361 may induce anti-inflammatory effects, while miR-133 may induce anti-apoptotic effects and hepatocyte proliferation in the ConA-treated mice that received Gal-9.

According to the results of the present study, miR-181 and miR-127 were significantly downregulated by Gal-9 administration in ConA-treated mice. It has been reported that miR-181 is increased during early $\mathrm{T}$ cell development and subsequently downregulated in mature CD4 T cells, which include Th1 and Th2 effector cells. Specifically, miR-181 functions to enhance T cell receptor (TCR) signaling strength by inhibiting multiple phosphatases that negatively regulate the TCR signaling cascade $(33,34)$. Downregulation of miR-181 also impairs TCR sensitivity by increasing dual specificity phosphatase 6 activity (35). In addition, miR-127 is downregulated by Gal-9, which facilitates hepatocyte proliferation in liver regeneration by releasing the pro-oncogene B-cell lymphoma 6 protein and SET domain-containing protein 8 (36). The reports indicate that downregulation of miR-181 and miR-127 by Gal-9 may induce anti-inflammatory effects and enhance hepatocyte proliferation.

In conclusion, the results of the present study demonstrated that Gal-9 ameliorated fulminant hepatitis, potentially by regulation of apoptosis and inflammation, stimulation of hepatocyte proliferation and alteration of the expression of miRNAs involved in these events. However, further studies are required to fully elucidate these mechanisms.

\section{Acknowledgements}

Dr Toshiro Niki and Dr Mitsuomi Hirashima are board members of GalPharma Co., Ltd (Takamatsu, Japan).
These two authors hold the following patent associated with material pertinent to this article: 'Novel modified galectin-9 proteins and use thereof', which was applied for by GalPharma Co., Ltd. and was issued in Japan (4792390), the USA (8268324), European Patent Office (EPO; 1736541), Canada (2561696), India (239130) and Korea (10-1222281) as of 2 December 2013. These two authors have the following products associated with material pertinent to this article: stable-form Gal-9. The authors thank Ms. Kayo Endo, Ms. Fuyuko Kokado, Ms. Keiko Fujikawa, Ms. Kayo Hirose, Dr Miwako Watanabe, Ms. Noriko Murao and Dr Kayo Ogawa, Department of Gastroenterology and Neurology, Faculty of Medicine, Kagawa University, for providing technical assistance.

\section{References}

1. Fujiwara K, Mochida S, Matsui A, Nakayama N, Nagoshi S and Toda G; Intractable Liver Diseases Study Group of Japan: Fulminant hepatitis and late onset hepatic failure in Japan. Hepatol Res 38: 646-657, 2008.

2. Kern M, Popov A, Scholz K, Schumak B, Djandji D, Limmer A, Eggle D, Sacher T, Zawatzky R, Holtappels R, et al: Virally infected mouse liver endothelial cells trigger CD8+ T-cell immunity. Gastroenterology 138: 336-346, 2010.

3. Fujiwara K, Yasui S, Yonemitsu Y, Arai M, Kanda T, Nakano M, Oda S and Yokosuka O: Fixed point observation of etiology of acute liver failure according to the novel Japanese diagnostic criteria. J Hepatobiliary Pancreat Sci 22: 225-229, 2015.

4. Ikegami T, Taketomi A, Soejima Y, Yoshizumi T, Sanefuji K, Kayashima H, Shimada M and Maehara Y: Living donor liver transplantation for acute liver failure: A 10-year experience in a single center. J Am Coll Surg 206: 412-418, 2008.

5. Saita N, Goto E, Yamamoto T, Cho I, Tsumori K, Kohrogi H, Maruo K, Ono T, Takeya M, Kashio Y, et al: Association of galectin-9 with eosinophil apoptosis. Int Arch Allergy Immunol 128: 42-50, 2002.

6. Zhu C, Anderson AC, Schubart A, Xiong H, Imitola J, Khoury SJ Zheng XX, Strom TB and Kuchroo VK: The Tim-3 ligand galectin- 9 negatively regulates $\mathrm{T}$ helper type 1 immunity. Nat Immunol 6: 1245-1252, 2005.

7. Oomizu S, Arikawa T, Niki T, Kadowaki T, Ueno M, Nishi N, Yamauchi A and Hirashima M: Galectin-9 suppresses Th17 cell development in an IL-2-dependent but Tim-3-independent manner. Clin Immunol 143: 51-58, 2012.

8. Seki M, Oomizu S, Sakata KM, Sakata A, Arikawa T, Watanabe K, Ito K, Takeshita K, Niki T, Saita N, et al: Galectin-9 suppresses the generation of Th17, promotes the induction of regulatory $\mathrm{T}$ cells, and regulates experimental autoimmune arthritis. Clin Immunol 127: 78-88, 2008.

9. Niki T, Tsutsui S, Hirose S, Aradono S, Sugimoto Y, Takeshita K, Nishi N and Hirashima M: Galectin-9 is a high affinity IgE-binding lectin with anti-allergic effect by blocking IgE-antigen complex formation. J Biol Chem 284: 32344-32352, 2009.

10. Kageshita T, Kashio Y, Yamauchi A, Seki M, Abedin MJ, Nishi N, Shoji H, Nakamura T, Ono T and Hirashima M: Possible role of galectin-9 in cell aggregation and apoptosis of human melanoma cell lines and its clinical significance. Int J Cancer 99: 809-816, 2002.

11. Zamore PD and Haley B: Ribo-gnome: The big world of small RNAs. Science 309: 1519-1524, 2005.

12. Chen JF, Mandel EM, Thomson JM, Wu Q, Callis TE, Hammond SM, Conlon FL and Wang DZ: The role of microRNA-1 and microRNA-133 in skeletal muscle proliferation and differentiation. Nat Genet 38: 228-233, 2006.

13. Bala S, Marcos M and Szabo G: Emerging role of microRNAs in liver diseases. World J Gastroenterol 15: 5633-5640, 2009.

14. Miyoshi H, Kato K, Iwama H, Maeda E, Sakamoto T, Fujita K, Toyota Y, Tani J, Nomura T, Mimura S, et al: Effect of the anti-diabetic drug metformin in hepatocellular carcinoma in vitro and in vivo. Int J Oncol 45: 322-332, 2014.

15. Morishita A and Masaki T: miRNA in hepatocellular carcinoma. Hepatol Res 45: 128-141, 2015. 
16. Japan Society of Clinical Chemistry, 'Recommendation for measuring enzyme activity in human serum. Lactate dehydrogenase (1989-08-30).' Japanese Journal of Clinical Chemistry 19: 228-246, 1990

17. Cortez KJ and Kottilil S: Beyond interferon: Rationale and prospects for newer treatment paradigms for chronic hepatitis $\mathrm{C}$. Ther Adv Chronic Dis 6: 4-14, 2015.

18. European Association For The Study Of The Liver: EASL clinical practice guidelines: Management of chronic hepatitis B virus infection. J Hepatol 57: 167-185, 2012.

19. European Association for Study of Liver: EASL clinical practice guidelines: Management of hepatitis $\mathrm{C}$ virus infection. J Hepatol 60: 392-420, 2014.

20. Bockmann JH, Dandri M, Lüth S, Pannicke N and Lohse AW: Combined glucocorticoid and antiviral therapy of hepatitis B virus-related liver failure. World J Gastroenterol 21: 2214-2219, 2015.

21. Sabatos CA, Chakravarti S, Cha E, Schubart A, Sánchez-Fueyo A, Zheng XX, Coyle AJ, Strom TB, Freeman GJ and Kuchroo VK Interaction of Tim-3 and Tim-3 ligand regulates T helper type 1 responses and induction of peripheral tolerance. Nat Immunol 4: 1102-1110, 2003.

22. Lv K, Zhang Y, Zhang M, Zhong $M$ and Suo Q: Galectin-9 ameliorates Con A-induced hepatitis by inducing CD4(+) CD25(low/int) effector T-Cell apoptosis and increasing regulatory T cell number. PLoS One 7: e48379, 2012.

23. Uchida Y, Ke B, Freitas MC, Yagita H, Akiba H, Busuttil RW, Najafian $\mathrm{N}$ and Kupiec-Weglinski JW: T-cell immunoglobulin mucin-3 determines severity of liver ischemia/reperfusion injury in mice in a TLR4-dependent manner. Gastroenterology 139: 2195-2206, 2010.

24. Hirao H, Uchida Y, Kadono K, Tanaka H, Niki T, Yamauchi A, Hata K, Watanabe T, Terajima $\mathrm{H}$ and Uemoto $\mathrm{S}$ : The protective function of galectin-9 in liver ischemia and reperfusion injury in mice. Liver Transpl 21: 969-981, 2015.

25. Mita E, Hayashi N, Iio S, Takehara T, Hijioka T, Kasahara A, Fusamoto $\mathrm{H}$ and Kamada T: Role of Fas ligand in apoptosis induced by hepatitis $\mathrm{C}$ virus infection. Biochem Biophys Res Commun 204: 468-474, 1994.

26. Feldstein AE, Canbay A, Angulo P, Taniai M, Burgart LJ, Lindor KD and Gores GJ: Hepatocyte apoptosis and fas expression are prominent features of human nonalcoholic steatohepatitis. Gastroenterology 125: 437-443, 2003.
27. Fujita K, Iwama H, Sakamoto T, Okura R, Kobayashi K, Takano J, Katsura A, Tatsuta M, Maeda E, Mimura S, et al: Galectin-9 suppresses the growth of hepatocellular carcinoma via apoptosis in vitro and in vivo. Int J Oncol 46: 2419-2430, 2015.

28. Kobayashi K, Morishita A, Iwama H, Fujita K, Okura R, Fujihara S, Yamashita T, Fujimori T, Kato K, Kamada H, et al: Galectin-9 suppresses cholangiocarcinoma cell proliferation by inducing apoptosis but not cell cycle arrest. Oncol Rep 34: 1761-1770, 2015

29. Kanitz A, Imig J, Dziunycz PJ, Primorac A, Galgano A, Hofbauer GF, Gerber AP and Detmar M: The expression levels of microRNA-361-5p and its target VEGFA are inversely correlated in human cutaneous squamous cell carcinoma. PLoS One 7: e49568, 2012.

30. Wang HW, Lo HH, Chiu YL, Chang SJ, Huang PH, Liao KH, Tasi CF, Wu CH, Tsai TN, Cheng CC and Cheng SM: Dysregulated miR-361-5p/VEGF axis in the plasma and endothelial progenitor cells of patients with coronary artery disease. PLoS One 9: e98070, 2014.

31. Haywood L, McWilliams DF, Pearson CI, Gill SE, Ganesan A, Wilson D and Walsh DA: Inflammation and angiogenesis in osteoarthritis. Arthritis Rheum 48: 2173-2177, 2003.

32. Xu C, Lu Y, Pan Z, Chu W, Luo X, Lin H, Xiao J, Shan H, Wang $\mathrm{Z}$ and Yang B: The muscle-specific microRNAs miR-1 and miR-133 produce opposing effects on apoptosis by targeting HSP60, HSP70 and caspase-9 in cardiomyocytes. J Cell Sci 120: 3045-3052, 2007

33. Li QJ, Chau J, Ebert PJ, Sylvester G, Min H, Liu G, Braich R, Manoharan M, Soutschek J, Skare P, et al: miR-181a is an intrinsic modulator of T cell sensitivity and selection. Cell 129: 147-161, 2007.

34. Raisch J, Darfeuille-Michaud A and Nguyen HT: Role of microRNAs in the immune system, inflammation and cancer. World J Gastroenterol 19: 2985-2996, 2013.

35. Li G, Yu M, Lee WW, Tsang M, Krishnan E, Weyand CM and Goronzy JJ: Decline in miR-181a expression with age impairs $\mathrm{T}$ cell receptor sensitivity by increasing DUSP6 activity. Nat Med 18: 1518-1524, 2012

36. Pan C, Chen H, Wang L, Yang S, Fu H, Zheng Y, Miao M and Jiao B: Down-regulation of MiR-127 facilitates hepatocyte proliferation during rat liver regeneration. PLoS One 7: e39151, 2012. 